\title{
A study on degree of approximation by Karamata summability method
}

\author{
Hare Krishna Nigam and Kusum Sharma*
}

\author{
* Correspondence: \\ kusum31sharma@rediffmail.com \\ Department of Mathematics, \\ Faculty of Engineering and \\ Technology, Mody Institute of \\ Technology and Science (Deemed \\ University), Laxmangarh-332311, \\ Sikar, Rajasthan, India
}

\begin{abstract}
Vuĉkoviĉ [Maths. Zeitchr. 89, 192 (1965)] and Kathal [Riv. Math. Univ. Parma, Italy 10, 3338 (1969)] have studied summability of Fourier series by Karamata $\left(K^{\lambda}\right)$ summability method. In present paper, for the first time, we study the degree of approximation of function $f \in \operatorname{Lip}(\alpha, r)$ and $f \in W\left(L_{r} \xi(t)\right)$ by $K^{\lambda}$-summability means of its Fourier series and conjugate of function $\tilde{f} \in \operatorname{Lip}(\alpha, r)$ and $\tilde{f} \in W\left(L_{r}, \xi(t)\right)$ by $K^{\lambda}$-summability means of its conjugate Fourier series and establish four quite new theorems.

MSC: primary 42B05; 42B08; 42A42; 42A30; 42A50.
\end{abstract}

Keywords: degree of approximation, $\operatorname{Lip}(a, r)$ class, $W\left(L_{r}, \xi(t)\right)$ class of functions, Fourier series, conjugate Fourier series, $K^{\lambda}$-summability, Lebesgue integral

\section{Introduction}

The method $K^{\lambda}$ was first introduced by Karamata [1] and Lotosky [2] reintroduced the special case $\lambda=1$. Only after the study of Agnew [3], an intensive study of these and similar cases took place. Vuckkoviĉ [4] applied this method for summability of Fourier series. Kathal [5] extended the result of Vuckkoviĉ [4]. Working in the same direction, Ojha [6], Tripathi and Lal [7] have studied $K^{\lambda}$-summability of Fourier series under different conditions. The degree of approximation of a function $f \in \operatorname{Lip} \alpha$ by Cesàro and Nörlund means of the Fourier series has been studied by Alexits [8], Sahney and Goel [9], Chandra [10], Qureshi [11], Qureshi and Neha [12], Rhoades [13], etc. But nothing seems to have been done so far in the direction of present work. Therefore, in present paper, we establish two new theorems on degree of approximation of function $f$ belonging to $\operatorname{Lip}(\alpha, r)(r \geq 1)$ and to weighted class $W\left(L_{r}, \xi(t)\right)(r \geq 1)$ by $K^{\lambda}$-means on its Fourier series and two other new theorems on degree of approximation of function $\tilde{f}$, conjugate of a $2 \pi$-periodic function $f$ belonging to Lip $(\alpha, r)(r>1)$ and to weighted class $W\left(L_{r}, \xi(t)\right)(r \geq 1)$ by $K^{\lambda}$-means on its conjugate Fourier series.

\section{Definitions and notations}

Let us define, for $n=0,1,2, \ldots$, the numbers $\left[\begin{array}{c}n \\ m\end{array}\right]$, for $0 \leq m \leq n$, by

$$
\begin{aligned}
\prod_{v-0}^{n-1}(x+v) & =\sum_{m=0}^{n}\left[\begin{array}{l}
n \\
m
\end{array}\right] x^{m}=\frac{\Gamma(x+n)}{\Gamma(x)} \\
& =x(x+1)(x+2) \ldots(x+n-1) .
\end{aligned}
$$


The numbers $\left[\begin{array}{c}n \\ m\end{array}\right]$ are known as the absolute value of stirling number of first kind Let $\left\{s_{n}\right\}$ be the sequence of partial sums of an infinite series $\Sigma u_{n}$, and let us write

$$
s_{n}^{\lambda}=\frac{\Gamma(\lambda)}{\Gamma(\lambda+n)} \sum_{m=0}^{n}\left[\begin{array}{l}
n \\
m
\end{array}\right] \lambda^{m} s_{m}
$$

to denote the $n$th $K^{\lambda}$-mean of order $\lambda>0$. If $s_{n}^{\lambda} \rightarrow s$ as $n \rightarrow \infty$, where $s$ is a fixed finite number, then the sequence $\left\{s_{n}\right\}$ or the series $\sum u_{n}$ is said to be summable by Karamata method $\left(K^{\lambda}\right)$ of order $\lambda>0$ to the sum $s$, and we can write

$$
s_{n}^{\lambda} \rightarrow s\left(K^{\lambda}\right) \quad \text { as } n \rightarrow \infty .
$$

Let $f$ be a $2 \pi$-periodic function and integrable in the sense of Lebesgue. The Fourier series associated with $f$ at a point $x$ is defined by

$$
f(x) \sim \frac{a_{0}}{2}+\sum_{n=1}^{\infty}\left(a_{n} \cos n x+b_{n} \sin n x\right) \equiv \sum_{n=1}^{\infty} A_{n}(x)
$$

with $n$th partial sums $s_{n}(f ; x)$.

The conjugate series of Fourier series (2.4) is given by

$$
\sum_{n=1}^{\infty}\left(a_{n} \sin n x-b_{n} \cos n x\right) \equiv \sum_{n=1}^{\infty} B_{n}(x)
$$

with $n$th partial sums $\tilde{s}_{n}(f ; x)$.

Throughout this paper, we will call (2.5) as conjugate Fourier series of function $f$.

$L_{\infty}$-norm of a function $f: R \rightarrow R$ is defined by

$$
\|f\|_{\infty}=\sup \{|f(x)|: x \in R\}
$$

$L_{r}$-norm is defined by

$$
\|f\|_{r}=\left(\int_{0}^{2 \pi}|f(x)|^{r} d x\right)^{\frac{1}{r}}, \quad r \geq 1 .
$$

The degree of approximation of a function $f: R \rightarrow R$ by a trigonometric polynomial $t_{n}$ of degree $n$ under sup norm \|\|$_{\infty}$ is defined by

(Zygmund [14])

$$
\left\|t_{n}-f\right\|_{\infty}=\sup \left\{\left|t_{n}(x)-f(x)\right|: x \in R\right\}
$$

and $E_{n}(f)$ of a function $f \in L_{r}$ is given by

$$
E_{n}(f)=\min _{t_{n}}\left\|t_{n}-f\right\|_{r} .
$$

This method of approximation is called trigonometric Fourier approximation. A function $f \in \operatorname{Lip} \alpha$ if

$$
|f(x+t)-f(x)|=O\left(|t|^{\alpha}\right) \quad \text { for } \quad 0<\alpha \leq 1
$$

and 
$f \in \operatorname{Lip}(\alpha, r)$ for $0 \leq x \leq 2 \pi$, if

$$
\left(\int_{0}^{2 \pi}|f(x+t)-f(x)|^{r} d x\right)^{\frac{1}{r}}=O\left(|t|^{\alpha}\right), \quad 0<\alpha \leq 1 \text { and } r \geq 1
$$

(definition 5.38 of McFadden [15]).

Given a positive increasing function $\xi(t)$ and an integer $r \geq 1, f \in \operatorname{Lip}(\xi(t), r)$, if

$$
\left(\int_{0}^{2 \pi}|f(x+t)-f(x)|^{r} d x\right)^{\frac{1}{r}}=O(\xi(t))
$$

and that

$f \in W\left(L_{r}, \xi(t)\right)$ if

$$
\left(\int_{0}^{2 \pi}\left|\{f(x+t)-f(x)\} \sin ^{\beta} x\right|^{r} d x\right)^{\frac{1}{r}}=O(\mid \xi(t)), \quad \beta \geq 0
$$

If $\beta=0$, our newly defined weighted i.e. $W\left(L_{r}, \xi(t)\right)$ reduces to $\operatorname{Lip}(\xi(t), r)$, if $\xi(t)=t^{\alpha}$ then $\operatorname{Lip}(\xi(t), r)$ coincides with $\operatorname{Lip}(\alpha, r)$ and if $r \rightarrow \infty$ then Lip $(\alpha, r)$ reduces to Lip $\alpha$.

We observe that

$$
\operatorname{Lip} \alpha \subseteq \operatorname{Lip}(\alpha, r) \subseteq \operatorname{Lip}(\xi(t), r) \subseteq W\left(L_{r}, \xi(t)\right) \quad \text { for } 0<\alpha \leq 1, r \geq 1 .
$$

We write

$$
\begin{aligned}
& \phi(t)=f(x+t)+f(x-t)-2 f(x) \\
& K_{n}(t)=\frac{\sum_{m=0}^{n}\left[\begin{array}{l}
n \\
m
\end{array}\right] \lambda^{m} \sin \left(m+\frac{1}{2}\right) t}{\Gamma(\lambda+n) \sin \left(\frac{t}{2}\right)} \\
& \psi(t)=f(x+t)-f(x-t) \\
& \tilde{K}_{n}(t)=\frac{\sum_{m=0}^{n}\left[\begin{array}{c}
n \\
m
\end{array}\right] \lambda^{m} \cos \left(m+\frac{1}{2}\right) t}{\Gamma(\lambda+n) \sin \left(\frac{t}{2}\right)} \\
& \tilde{f}(x)=-\frac{1}{2 \pi} \int_{0}^{\pi} \psi(t) \cot \left(\frac{t}{2}\right) d t
\end{aligned}
$$

\section{The main results}

\subsection{Theorem 1}

If a function $f, 2 \pi$-periodic, belonging to $\operatorname{Lip}(\alpha, r)$ then its degree of approximation by $K^{\lambda}$-summability means on its Fourier series is given by

$$
\begin{array}{r}
\left\|s_{n}-f\right\|_{r}=O\left[\frac{1}{(n+1)^{\alpha-\frac{1}{r}}}\left\{\frac{\log (n+1) e}{(n+1)}\right\}+\frac{1}{\Gamma(\lambda+n)}\right], \\
0<\alpha \leq 1, n=0,1,2 \ldots,
\end{array}
$$

where $s_{n}$ is $K^{\lambda}$-mean of Fourier series (2.4).

\subsection{Theorem 2}

If a function $f, 2 \pi$-periodic, belonging to $W\left(L_{r}, \xi(t)\right)$ then its degree of approximation by $K^{\lambda}$-summability means on its Fourier series is given by 


$$
\left\|s_{n}-f\right\|_{r}=O\left[\left\{(n+1)^{\beta+\frac{1}{r}} \xi\left(\frac{1}{n+1}\right)\right\}\left\{\frac{\log (n+1)}{(n+1)}+\frac{1}{(n+1)^{2}}+\frac{1}{\Gamma(\lambda+n)}\right\}\right]
$$

provided that $\xi(t)$ satisfies the following conditions:

$$
\begin{aligned}
& \left\{\frac{\xi(t)}{t}\right\} \text { is non - increasing in } t \\
& \left\{\int_{0}^{\frac{1}{n+1}}\left(\frac{t|\phi(t)|}{\xi(t)}\right)^{r} \sin ^{\beta r} t d t\right\}^{\frac{1}{r}}=O\left(\frac{1}{n+1}\right),
\end{aligned}
$$

and

$$
\left\{\int_{\frac{1}{n+1}}^{\pi}\left(\frac{t^{-\delta}|\phi(t)|}{\xi(t)}\right)^{r} d t\right\}^{\frac{1}{r}}=O\left((n+1)^{\delta}\right)
$$

where $\delta$ is an arbitrary positive number such that $s(1-\delta)-1>0, \frac{1}{r}+\frac{1}{s}=1,1 \leq r \leq$ $\infty$, conditions (3.4) and (3.5) hold uniformly in $x, s_{n}$ is $K^{\lambda}$-mean of Fourier series (2.4).

\subsection{Theorem 3}

If a function $\tilde{f}$, conjugate to a $2 \pi$-periodic function $f$, belonging to $\operatorname{Lip}(\alpha, r)$ then its degree of approximation by $K^{\lambda}$-summability means on its conjugate Fourier series is given by

$$
\begin{array}{r}
\left\|\tilde{s}_{n}-\tilde{f}\right\|_{r}=O\left[\frac{1}{(n+1)^{\alpha-\frac{1}{r}}}\left\{\frac{\log (n+1) e}{(n+1)^{2}}\right\}+\frac{1}{\Gamma(\lambda+n)}+1\right], \\
0<\alpha \leq 1, n=0,1,2, \ldots,
\end{array}
$$

where $\tilde{s}_{n}$ is $K^{\lambda}$-mean of conjugate Fourier series (2.5) and

$$
\tilde{f}(x)=-\frac{1}{2 \pi} \int_{0}^{\pi} \psi(t) \cot \left(\frac{t}{2}\right) d t
$$

\subsection{Theorem 4}

If a function $\tilde{f}$, conjugate to a $2 \pi$-periodic function $f$, belonging to $W\left(L_{n} \xi(t)\right)$ then its degree of approximation by $K^{\lambda}$-summability means on its conjugate Fourier series is given by

$$
\left\|\tilde{s}_{n}-\tilde{f}\right\|_{r}=O\left\{(n+1)^{\beta+\frac{1}{r}} \xi\left(\frac{1}{n+1}\right)\right\}\left[\frac{2}{(n+1)^{2}}+\frac{\log (n+1)}{(n+1)^{2}}+\frac{1}{\Gamma(\lambda+n)}\right]
$$

provided that $\xi(t)$ satisfies the conditions (3.3)-(3.5) in which $\delta$ is an arbitrary positive number such that $s(1-\delta)-1>0, \frac{1}{r}+\frac{1}{s}=1,1 \leq r \leq \infty$. Conditions (3.4) and (3.5) hold uniformly in $x, \tilde{s}_{n}$ is $K^{\lambda}$-mean of conjugate Fourier series (2.5) and

$$
\tilde{f}(x)=-\frac{1}{2 \pi} \int_{0}^{\pi} \psi(t) \cot \left(\frac{t}{2}\right) d t
$$

\section{Lemmas}

For the proof of our theorems, following lemmas are required. 


\subsection{Lemma 1}

(Vuĉkoviĉ [14]). Let $\lambda>0$ and $0<t<\frac{\pi}{2}$, then

$$
\frac{\operatorname{Im} \Gamma\left(\lambda e^{i t}+n\right)}{\Gamma(\lambda \cos t+n) \sin \left(\frac{t}{2}\right)}=\frac{|\sin (\lambda \log (n+1) \cdot \sin t)|}{\sin \left(\frac{t}{2}\right)}+O(1) \quad \text { as } n \rightarrow \infty \text { uniformly in } t .
$$

\subsection{Lemma 2}

$$
K_{n}(t)=O\{\lambda \log (n+1)\}+O(1) .
$$

Proof. For $0<t<\frac{1}{n+1}, 1-\cos t<\frac{t^{2}}{2}$, sin $n t \leq n t$ and $\sin \frac{t}{2} \geq \frac{t}{\pi}$

$$
\begin{aligned}
& \left|K_{n}(t)\right| \leq\left|\frac{1}{\Gamma(\lambda+n)} \sum_{m=0}^{n}\left[\begin{array}{c}
n \\
m
\end{array}\right] \cdot \lambda^{m} \frac{\sin \left(m+\frac{1}{2}\right) t}{\sin \frac{t}{2}}\right| \\
& =O\left[\frac{\operatorname{Im}\left\{e^{\left.\frac{i t}{2} \frac{\Gamma\left(\lambda e^{i t}+n\right)}{\Gamma\left(\lambda e^{i t}\right)}\right\}}\right.}{\Gamma(\lambda+n) \sin \frac{t}{2}}\right] \text { by (2.1) } \\
& =O\left[\frac{\operatorname{Im} \Gamma\left(\lambda e^{i t}+n\right)}{\Gamma(\lambda+n) \sin \frac{t}{2}}\right]+O\left[\frac{\operatorname{Re} \Gamma\left(\lambda e^{i t}+n\right)}{\Gamma(\lambda+n)}\right] \\
& =O\left[\frac{\Gamma(\lambda \cos t+n)}{\Gamma(\lambda+n)} \cdot \frac{\operatorname{Im} \Gamma\left(\lambda e^{i t}+n\right)}{\Gamma(\lambda \cos t+n) \sin \frac{t}{2}}\right]+O\left[\frac{\Gamma(\lambda \cos t+n)}{\Gamma(\lambda+n)}\right] \\
& =O\left[n^{-\lambda(1-\cos t)} \cdot \frac{\operatorname{Im} \Gamma\left(\lambda e^{i t}+n\right)}{\Gamma(\lambda \cos t+n) \sin \frac{t}{2}}\right]+O\left[n^{-\lambda(1-\cos t)}\right] \\
& =O\left[e^{-\lambda(1-\cos t) \log n} \cdot \frac{\operatorname{Im} \Gamma\left(\lambda e^{i t}+n\right)}{\Gamma(\lambda \cos t+n) \sin \frac{t}{2}}\right]+O\left[e^{-\lambda(1-\cos t) \log n}\right] \\
& =O\left[e^{-\frac{\lambda}{2} t^{2} \log (n+1)} \cdot \frac{\operatorname{Im} \Gamma\left(\lambda e^{i t}+n\right)}{\Gamma(\lambda \cos t+n) \sin \frac{t}{2}}\right]+O\left[e^{-\frac{\lambda}{2} t^{2} \log (n+1)}\right] .
\end{aligned}
$$

Considering first part of (4.1) and using Lemma 1,

$$
\begin{aligned}
K_{n}(t)= & O\left[e^{-\frac{\lambda}{2} t^{2} \log (n+1)} \cdot \frac{|\sin (\lambda \log (n+1) \cdot \sin t)|}{\sin \left(\frac{t}{2}\right)}\right]+O\left[e^{-\frac{\lambda}{2} t^{2} \log (n+1)}\right] \\
& +O\left[e^{-\frac{\lambda}{2} t^{2} \log (n+1)}\right] \\
= & O\left[e^{-\frac{\lambda}{2} t^{2} \log (n+1)} \cdot \frac{|\sin (\lambda \log (n+1) \cdot \sin t)|}{\sin \left(\frac{t}{2}\right)}\right]+O\left[e^{-\frac{\lambda}{2} t^{2} \log (n+1)}\right] \\
= & O\{\lambda \log (n+1)\}\left[\frac{|\sin (\lambda \log (n+1) \cdot \sin t)|}{\sin \left(\frac{t}{2}\right)}\right]+O(1) \\
= & O\{\lambda \log (n+1)\}+O(1) .
\end{aligned}
$$




\subsection{Lemma 3}

$$
\begin{aligned}
\tilde{K}_{n}(t)= & O\left[\frac{e^{-\frac{\lambda}{2} t^{2} \log (n+1)}}{t}\right]+O\{\lambda \log (n+1)\}|\sin (\lambda \log (n+1) \cdot \sin t)| \\
& +O\left[e^{-\frac{\lambda}{2} t^{2} \log (n+1)} \cdot|\sin (t / 2)|\right] .
\end{aligned}
$$

Proof. For $0<t<\frac{1}{n+1}, 1-\cos t<\frac{t^{2}}{2}, \sin n t \leq n t$ and $\sin \frac{t}{2} \geq \frac{t}{\pi}$

$$
\begin{aligned}
& \left|K_{n}(t)\right| \leq\left|\frac{1}{\Gamma(\lambda+n)} \sum_{m=0}^{n}\left[\begin{array}{c}
n \\
m
\end{array}\right] \cdot \lambda^{m} \frac{\cos \left(m+\frac{1}{2}\right) t}{\sin \frac{t}{2}}\right| \\
& =O\left[\frac{\operatorname{Re}\left\{e^{\left.\frac{i t}{2} \frac{\Gamma\left(\lambda e^{i t}+n\right)}{\Gamma\left(\lambda e^{i t}\right)}\right\}}\right.}{\Gamma(\lambda+n) \sin \frac{t}{2}}\right] \text { by (2.1) } \\
& =O\left[\frac{\operatorname{Re} \Gamma\left(\lambda e^{i t}+n\right)}{\Gamma(\lambda+n) \sin \frac{t}{2}}\right]+O\left[\frac{\operatorname{Im} \Gamma\left(\lambda e^{i t}+n\right)}{\Gamma(\lambda+n)}\right] \\
& =O\left[\frac{\Gamma(\lambda \cos t+n)}{\Gamma(\lambda+n) \sin \frac{t}{2}}\right]+O\left[\frac{\Gamma(\lambda \cos t+n)}{\Gamma(\lambda+n)} \cdot \frac{\operatorname{Im} \Gamma\left(\lambda e^{i t}+n\right)}{\Gamma(\lambda \cos t+n)}\right] \\
& =O\left[\frac{n^{-\lambda(1-\cos t)}}{\sin \frac{t}{2}}\right]+O\left[n^{-\lambda(1-\cos t)} \cdot \frac{\operatorname{Im} \Gamma\left(\lambda e^{i t}+n\right)}{\Gamma(\lambda \cos t+n)}\right] \\
& =O\left[\frac{e^{-\lambda(1-\cos t) \log n}}{\sin \frac{t}{2}}\right]+O\left[e^{-\lambda(1-\cos t) \log n} \cdot \frac{\operatorname{Im} \Gamma\left(\lambda e^{i t}+n\right)}{\Gamma(\lambda \cos t+n)}\right] \\
& =O\left[\frac{e^{-\frac{\lambda}{2} t^{2} \log n}}{\sin \frac{t}{2}}\right]+O\left[e^{-\frac{\lambda}{2} t^{2} \log n} \cdot \frac{\operatorname{Im} \Gamma\left(\lambda e^{i t}+n\right)}{\Gamma(\lambda \cos t+n)}\right] \\
& =O\left[\frac{e^{-\frac{\lambda}{2} t^{2} \log (n+1)}}{t}\right]+O\left[e^{-\frac{\lambda}{2} t^{2} \log (n+1)} \cdot \frac{\operatorname{Im} \Gamma\left(\lambda e^{i t}+n\right)}{\Gamma(\lambda \cos t+n)}\right] \text {. }
\end{aligned}
$$

Using Lemma 1,

$$
\begin{aligned}
K_{n}(t)= & O\left[\frac{e^{-\frac{\lambda}{2} t^{2} \log (n+1)}}{t}\right]+O\left[e^{-\frac{\lambda}{2} t^{2} \log (n+1)} \cdot|\sin (\lambda \log (n+1) \cdot \sin t)|\right] \\
& +O\left[e^{-\frac{\lambda}{2} t^{2} \log (n+1)} \cdot|\sin (t / 2)|\right] \\
= & O\left[\frac{e^{-\frac{\lambda}{2} t^{2} \log (n+1)}}{t}\right]+O\{\lambda \log (n+1)\}|\sin (\lambda \log (n+1) \cdot \sin t)| \\
& +O\left[e^{-\frac{\lambda}{2} t^{2} \log (n+1)} \cdot|\sin (t / 2)|\right] .
\end{aligned}
$$




\subsection{Lemma 4}

(McFadden [15]), Lemma 5.40) If $f(x)$ belongs to $\operatorname{Lip}(\alpha, r)$ on $[0, \pi]$, then $\phi(t)$ belongs to $\operatorname{Lip}(\alpha, r)$ on $[0, \pi]$.

\section{Proof of the theorems}

\subsection{Proof of Theorem 1}

Following Titchmarsh [16] and using Riemann-Lebesgue theorem, the $m$ th partial sum $s_{m}(x)$ of series (2.4) at $t=x$ is given by

$$
s_{m}(x)-f(x)=\frac{1}{2 \pi} \int_{0}^{\pi} \phi(t) \frac{\sin \left(m+\frac{1}{2}\right) t}{\sin \frac{t}{2}} d t
$$

Therefore,

$$
\begin{aligned}
\frac{\Gamma(\lambda)}{\Gamma(\lambda+n)} \sum_{m=0}^{n}\left[\begin{array}{c}
n \\
m
\end{array}\right] \lambda^{m}\left\{s_{m}(x)-f(x)\right\}= & \frac{1}{2 \pi} \int_{0}^{\pi} \phi(t) \frac{\Gamma(\lambda)}{\Gamma(\lambda+n)} \sum_{m+0}^{n}\left[\begin{array}{c}
n \\
m
\end{array}\right] \\
& \cdot \lambda^{m} \frac{\sin \left(m+\frac{1}{2}\right) t}{\sin \frac{t}{2}} d t \\
s_{m}(x)-f(x)= & \frac{\Gamma(\lambda)}{2 \pi} \int_{0}^{\pi} \phi(t) K_{n}(t) d t \\
= & \frac{\Gamma(\lambda)}{2 \pi}\left[\int_{0}^{\frac{1}{n+1}+\int_{\frac{1}{n+1}}^{\pi}}\right] \phi(t) K_{n}(t) d t \\
= & O\left(I_{1.1}\right)+O\left(I_{1.2}\right) \text { (say). }
\end{aligned}
$$

Now we consider,

$$
I_{1.1}=\int_{0}^{\frac{1}{n+1}}\left|\phi(t) \| K_{n}(t)\right| d t .
$$

Using Lemma 2,

$$
I_{1.1}=O\{\lambda \log (n+1)\} \int_{0}^{\frac{1}{n+1}}|\phi(t)| d t+O\left[\int_{0}^{\frac{1}{n+1}}|\phi(t)| d t\right] .
$$

Using Hölder's inequality and Lemma 4,

$$
\begin{aligned}
I_{1.1}= & O[\{\lambda \log (n+1)\}+1]\left[\int_{0}^{\frac{1}{n+1}}\left\{\frac{t \phi(t)}{t^{\alpha}}\right\}^{r} d t\right]^{\frac{1}{r}}\left[\int_{0}^{\frac{1}{n+1}}\left(t^{\alpha-1}\right)^{s} d t\right]^{\frac{1}{s}} \\
& =O[\{\lambda \log (n+1)\}+1]\left(\frac{1}{n+1}\right)\left[\left\{\frac{t^{\alpha s-s+1}}{\alpha s-s+1}\right\}_{0}^{\left.\frac{1}{n+1}\right]^{\frac{1}{s}}}\right]^{\frac{1}{s}} \\
= & \left.O\left\{\frac{\log (n+1) e}{(n+1)}\right\}\left[\frac{1}{(n+1)^{\alpha s-s+1}}\right]^{\frac{1}{s}}\right] \\
= & O\left[\left\{\frac{\log (n+1) e}{(n+1)}\right\} \frac{(n+1)^{\alpha-1+\frac{1}{s}}}{{ }^{\alpha}}\right] \\
= & O\left[\left\{\frac{\log (n+1) e}{(n+1)}\right\}\left\{\frac{1}{(n+1)^{\alpha-\frac{1}{r}}}\right\}\right] \text { since } \frac{1}{r}+\frac{1}{s}=1 .
\end{aligned}
$$


Since, for $\frac{1}{n+1} \leq t \leq \pi, \sin \frac{t}{2} \geq \frac{t}{\pi}$

$$
\begin{aligned}
K_{n}(t) & =O\left[\frac{1}{\Gamma(\lambda+n) \sin \frac{t}{2}}\right] \\
& =O\left[\frac{1}{\Gamma(\lambda+n) t}\right] .
\end{aligned}
$$

Next we consider,

$$
\left|I_{1.2}\right| \leq \int_{\frac{1}{n+1}}^{\pi}|\phi(t)|\left|K_{n}(t)\right| d t .
$$

Using Hölder's inequality, (5.3) and Lemma 4,

$$
\begin{aligned}
I_{1.2} & =O\left(\frac{1}{\Gamma(\lambda+n)}\right)\left[\int_{\frac{1}{n+1}}^{\pi}\left\{\frac{t^{-\delta} \phi(t)}{t^{\alpha}}\right\}^{r} d t\right]^{\frac{1}{r}}\left[\int_{\frac{1}{n+1}}^{\pi}\left(\frac{\delta^{\delta+\alpha}}{t}\right)^{s} d t\right]^{\frac{1}{s}} \\
& =O\left(\frac{1}{\Gamma(\lambda+n)}\right) \frac{1}{(n+1)^{-\delta}}\left[\int_{\left.\frac{1}{n+1} t^{(\delta+\alpha-1) s} d t\right]^{\frac{1}{s}}}\right. \\
& =O\left(\frac{1}{\Gamma(\lambda+n)}\right) \frac{1}{(n+1)^{-\delta}}\left[\left\{\frac{t^{(\delta+\alpha-1) s+1}}{(\delta+\alpha-1) s+1}\right\}_{\frac{1}{n+1}}^{\pi}\right]^{\frac{1}{s}} \\
& =O\left(\frac{1}{\Gamma(\lambda+n)}\right) \frac{1}{(n+1)^{-\delta}}\left[\frac{1}{(n+1)^{(\delta+\alpha-1) s+1}}\right]^{\frac{1}{s}} \\
& \left.=O\left(\frac{1}{\Gamma(\lambda+n)}\right) \frac{1}{(n+1)^{-\delta}}\left[\frac{1}{(n+1)^{(\delta+\alpha-1)+\frac{1}{s}}}\right]^{\frac{1}{\Gamma(\lambda+n)}}\right)\left[\frac{1}{(n+1)^{\alpha-1+\frac{1}{s}}}\right] \\
& =O\left(\frac{1}{(n+1)^{\alpha-\frac{1}{r}}}\right] .
\end{aligned}
$$

Combining (5.1), (5.2) and (5.4),

$$
\begin{aligned}
S_{m}-f(x) & =O\left[\left(\frac{\log (n+1) e}{(n+1)}\right)\left(\frac{1}{(n+1)^{\alpha-\frac{1}{r}}}\right)\right]+O\left[\left(\frac{1}{\Gamma(\lambda+n)}\right)\left(\frac{1}{(n+1)^{\alpha-\frac{1}{r}}}\right)\right] \\
& =O\left[\frac{1}{(n+1)^{\alpha-\frac{1}{r}}}\left\{\frac{\log (n+1) e}{(n+1)}+\frac{1}{\Gamma(\lambda+n)}\right\}\right] .
\end{aligned}
$$

This completes the proof of Theorem 1 . 


\subsection{Proof of Theorem 2}

Following the proof of Theorem 1,

$$
\begin{aligned}
S_{m}(x)-f(x) & =\frac{\Gamma(\lambda)}{2 \pi}\left[\int_{0}^{\frac{1}{n+1}}+\int_{\frac{1}{n+1}}^{\pi}\right] \phi(t) K_{n}(t) d t \\
& =O\left(I_{2.1}\right)+O\left(I_{2.2}\right) \quad \text { (say) } .
\end{aligned}
$$

We have

$$
|\phi(x+t)-\phi(x)| \leq|f(u+x+t)-f(u+x)|+|f(u-x-t)-f(u-x)| .
$$

Hence, by Minkowiski's inequality,

$$
\begin{aligned}
{\left[\left.\int_{0}^{2 \pi}\{\mid \phi(x+t)-\phi(x)\} \sin ^{\beta} x\right|^{r} d x\right]^{\frac{1}{r}} \leq } & {\left[\int_{0}^{2 \pi}\left|\{f(u+x+t)-f(u+x)\} \sin ^{\beta} x\right|^{r} d x\right]^{\frac{1}{r}} } \\
& +\left[\int_{0}^{2 \pi}\left|\{f(u-x-t)-f(u-x)\} \sin ^{\beta} x\right|^{r} d x\right]^{\frac{1}{r}} \\
= & O\{\xi(t)\} .
\end{aligned}
$$

Then $f \in W\left(L_{r}, \xi(t)\right) \Rightarrow \phi \in W\left(L_{r}, \xi(t)\right)$.

Now we consider,

$$
\left|I_{2.1}\right| \leq \int_{0}^{\frac{1}{n+1}}\left|\phi(t) \| K_{n}(t)\right| d t .
$$

Using Lemma 2,

$$
I_{2.1}=[O\{\lambda \log (n+1)\}+O(1)] \int_{0}^{\frac{1}{n+1}}|\phi(t)| d t .
$$

Using Hölder's inequality and the fact that $\phi(t) \in W\left(L_{r}, \xi(t)\right)$,

$$
\begin{aligned}
I_{2.1}=O & {[\{\lambda \log (n+1)\}+1]\left[\int_{0}^{\frac{1}{n+1}}\left\{\frac{t|\phi(t)| \sin ^{\beta}(t)}{\xi(t)}\right\}^{r} d t\right]^{\frac{1}{r}} } \\
& \cdot\left[\int_{0}^{\frac{1}{n+1}}\left\{\frac{\xi(t)}{t \sin ^{\beta} t}\right\}^{s} d t\right]^{\frac{1}{s}} \\
= & O\{\lambda \log (n+1) e\}\left(\frac{1}{n+1}\right)\left[\int_{0}^{\left.\frac{1}{n+1}\left\{\frac{\xi(t)}{t \sin ^{\beta} t}\right\}^{s} d t\right]^{\frac{1}{s}}}\right. \text { by }
\end{aligned}
$$


Since $\sin t \geq 2 t / \pi$,

$$
I_{2.1}=O\left(\frac{\log (n+1) e}{n+1}\right)\left[\int_{0}^{\frac{1}{n+1}}\left\{\frac{\xi(t)}{t^{1+\beta}}\right\}^{s} d t\right]^{\frac{1}{s}} .
$$

Since $\xi(t)$ is a positive increasing function and using second mean value theorem for integrals,

$$
\begin{aligned}
I_{2.1}= & O\left\{\left(\frac{\log (n+1) e}{n+1}\right) \xi\left(\frac{1}{n+1}\right)\right\}\left[\int_{\epsilon}^{\frac{1}{n+1}}\left\{\frac{1}{t^{(1+\beta) s}}\right\} d t\right]^{\frac{1}{s}} \text { for some } 0<\epsilon<\frac{1}{n+1} \\
= & O\left\{\left(\frac{\log (n+1) e}{n+1}\right) \xi\left(\frac{1}{n+1}\right)\right\}\left[\left\{\frac{t^{-(1+\beta) s+1}}{-(1+\beta) s+1}\right\}_{\epsilon}^{\frac{1}{n+1}}\right]^{\frac{1}{s}} \\
= & O\left[\left\{\left(\frac{\log (n+1) e}{n+1}\right) \xi\left(\frac{1}{n+1}\right)\right\}\left\{(n+1)^{(1+\beta)-\frac{1}{s}}\right\}\right] \\
= & O\left[\left\{\left(\frac{\log (n+1) e}{n+1}\right) \xi\left(\frac{1}{n+1}\right)\right\}\left\{(n+1)^{\beta+\frac{1}{r}}\right\}\right] \text { since } \frac{1}{r}+\frac{1}{s}=1 .
\end{aligned}
$$

Next we consider,

$$
\left|I_{2.2}\right| \leq \int_{\frac{1}{n+1}}^{\pi}|\phi(t)|\left|K_{n}(t)\right| d t
$$

Using Hölder's inequality, $|\sin t| \leq 1, \sin t \geq 2 t / \pi$, (5.3), conditions (3.3), (3.5) and second mean value theorem for integrals,

$$
\begin{aligned}
I_{2.2} & =O\left[\int_{\frac{1}{n+1}}^{\pi} \frac{1}{\Gamma(\lambda+n) t}|\phi(t)| d t\right] \\
& =O\left(\frac{1}{\Gamma(\lambda+n)}\right)\left[\int_{\frac{1}{n+1}}^{\pi}\left\{\frac{t^{-\delta}|\phi(t)| \sin ^{\beta}(t)}{\xi(t)}\right\}^{r} d t\right]^{\frac{1}{r}}\left[\int_{\frac{1}{n+1}}^{\pi}\left\{\frac{\xi(t)}{t^{-\delta} \sin ^{\beta} t t}\right\}^{s} d t\right]^{\frac{1}{s}} \\
& =O\left(\frac{1}{\Gamma(\lambda+n)}\right)\left[\int_{\frac{1}{n+1}}^{\pi}\left\{\frac{t^{-\delta}|\phi(t)|}{\xi(t)}\right\}^{r} d t\right]^{\frac{1}{r}}\left[\int_{\frac{1}{n+1}}^{\pi}\left\{\frac{\xi(t)}{t^{-\delta+\beta+1}}\right\}^{s} d t\right]^{\frac{1}{s}} \\
& =O\left[\left\{\frac{1}{\Gamma(\lambda+n)}\right\}\left\{(n+1)^{\delta}\right\}\right]\left[\int_{\frac{1}{n+1}}^{\pi}\left\{\frac{\xi(t)}{t^{-\delta+\beta+1}}\right\}^{s} d t\right]^{\frac{1}{s}} .
\end{aligned}
$$


Putting $t=\frac{1}{y}$

$$
\begin{aligned}
I_{2.2} & =O\left\{\frac{(n+1)^{\delta}}{\Gamma(\lambda+n)}\right\}\left[\int_{\pi}^{n+1}\left\{\frac{\xi\left(\frac{1}{y}\right)}{\gamma^{\delta-\beta-1^{\prime}}}\right\} \frac{d y}{\gamma^{2}}\right]^{\frac{1}{s}} \\
& =O\left\{\frac{(n+1)^{\delta}}{\Gamma(\lambda+n)} \xi\left(\frac{1}{n+1}\right)\right\}\left[\int_{\eta}^{n+1}\left\{\frac{d y}{d^{s(\delta-\beta-1)+2}}\right\} d t\right]^{\frac{1}{s}} \text { for some } \frac{1}{\pi} \leq \eta \leq n+1 \\
& =O\left\{\frac{(n+1)^{\delta}}{\Gamma(\lambda+n)} \xi\left(\frac{1}{n+1}\right)\right\}\left[\int_{1}^{n+1}\left\{\frac{d y}{\gamma^{s(\delta-\beta-1)+2}}\right\} d t\right]^{\frac{1}{s}} \text { for some } \frac{1}{\pi} \leq 1 \leq n+1 \\
& =O\left\{\frac{(n+1)^{\delta}}{\Gamma(\lambda+n)} \xi\left(\frac{1}{n+1}\right)\right\}\left[\left\{\frac{y^{s(\beta+1-\delta)-1}}{s(\beta+1-\delta)-1}\right\}_{1}^{n+1}\right]^{\frac{1}{s}} \\
& =O\left\{\frac{(n+1)^{\delta}}{\Gamma(\lambda+n)} \xi\left(\frac{1}{n+1}\right)\right\}\left\{(n+1)^{1+\beta-\delta-\frac{1}{s}}\right\} \\
& =O\left\{\frac{\xi\left(\frac{1}{n+1}\right)}{\Gamma(\lambda+n)}\right\}\left\{(n+1)^{\beta+\frac{1}{r}}\right\} \text { since } \frac{1}{r}+\frac{1}{s}=1 .
\end{aligned}
$$

Now combining (5.5)-(5.7),

$$
\begin{aligned}
\left|s_{m}(x)-f(x)\right|= & O\left[\left\{\left(\frac{\log (n+1) e}{(n+1)}\right) \xi\left(\frac{1}{n+1}\right)\right\}\left\{(n+1)^{\beta+\frac{1}{r}}\right\}\right] \\
& +O\left[\left\{\left(\frac{1}{\Gamma(\lambda+n)}\right) \xi\left(\frac{1}{n+1}\right)\right\}\left\{(n+1)^{\beta+\frac{1}{r}}\right\}\right] \\
= & O\left\{(n+1)^{\beta+\frac{1}{r}} \xi\left(\frac{1}{n+1}\right)\right\}\left[\frac{\log (n+1) e}{(n+1)}+\frac{1}{\Gamma(\lambda+n)}\right] .
\end{aligned}
$$

Now using $L_{r}$-norm, we get

$$
\begin{aligned}
\left\|s_{m}(x)-f(x)\right\|= & \left\{\int_{0}^{2 \pi}\left|s_{m}(x)-f(x)\right|^{r} d x\right\}^{\frac{1}{r}} \\
= & O\left[\int_{0}^{2 \pi}\left\{(n+1)^{\beta+\frac{1}{r}} \xi\left(\frac{1}{n+1}\right)\right\}\right. \\
& \left.\cdot\left\{\frac{\log (n+1) e}{(n+1)}+\frac{1}{\Gamma(\lambda+n)}\right\} d x\right]^{\frac{1}{r}} \\
= & {\left[\left\{(n+1)^{\beta+\frac{1}{r}} \xi\left(\frac{1}{n+1}\right)\right\}\right.} \\
& \left.\cdot\left\{\frac{\log (n+1) e}{(n+1)}+\frac{1}{\Gamma(\lambda+n)}\right\}\right]\left[\left\{\int_{0}^{2 \pi} d x\right\}^{\frac{1}{r}}\right] \\
= & O\left\{(n+1)^{\beta+\frac{1}{r}} \xi\left(\frac{1}{n+1}\right)\right\}\left[\frac{\log (n+1) e}{(n+1)}+\frac{1}{\Gamma(\lambda+n)}\right] .
\end{aligned}
$$

This completes the proof of Theorem 2 . 


\subsection{Proof of Theorem 3}

Following Lal [7], the $m$ th partial sum $\tilde{S}_{m}(x)$ of series (2.5) at $t=x$

$$
\tilde{S}_{m}(x)-\left[-\frac{1}{2 \pi} \int_{0}^{\pi} \psi(t) \cot \left(\frac{t}{2}\right) d t\right]=\frac{1}{2 \pi} \int_{0}^{\pi} \psi(t) \frac{\cos \left(m+\frac{1}{2}\right) t}{\sin \frac{t}{2}} d t .
$$

Therefore,

$$
\begin{aligned}
& \frac{\Gamma(\lambda)}{\Gamma(\lambda+n)} \sum_{m=0}^{n}\left[\begin{array}{c}
n \\
m
\end{array}\right] \lambda^{m}\left\{\tilde{S}(x)-\left(-\frac{1}{2 \pi} \int_{0}^{\pi} \psi(t) \cot \left(\frac{t}{2}\right) d t\right)\right\} \\
& =\frac{1}{2 \pi} \int_{0}^{\pi} \psi(t) \frac{\Gamma(\lambda)}{\Gamma(\lambda+n)} \sum_{m=0}^{n}\left[\begin{array}{c}
n \\
m
\end{array}\right] \lambda^{m} \frac{\cos \left(m+\frac{1}{2}\right) t}{\sin \frac{t}{2}} d t \\
\tilde{S}_{m}(x)-\tilde{f}(x) & =\frac{\Gamma(\lambda)}{2 \pi} \int_{0}^{\pi} \psi(t) \tilde{K}_{n}(t) d t \\
& =\frac{\Gamma(\lambda)}{2 \pi}\left[\int_{0}^{\frac{1}{n+1}}+\int_{\frac{1}{n+1}}^{\pi}\right]\left|\psi(t) \| \tilde{K}_{n}(t)\right| d t \\
& =O\left(I_{3.1}\right)+O\left(I_{3.2}\right) .
\end{aligned}
$$

We consider,

$$
\left|I_{3.1}\right|=\int_{0}^{\frac{1}{n+1}}|\psi(t)|\left|\tilde{K}_{n}(t)\right| d t .
$$

Using Lemma 3,

$$
\begin{aligned}
& I_{3.1}= O\left[\int_{0}^{\frac{1}{n+1}} \frac{e^{-\frac{\lambda}{2} t^{2} \log (n+1)}}{t}|\psi(t)| d t\right] \\
&+ O\{\lambda \log (n+1)\} \int_{0}^{\frac{1}{n+1}}|\sin (\lambda \log (n+1) \cdot \sin t) \| \psi(t)| d t \\
&+ O\left[\int_{0}^{\frac{1}{n+1}} e^{-\frac{\lambda}{2} t^{2} \log (n+1)}|\sin (t / 2) \| \psi(t)| d t\right] \\
&=I_{3.1 .1}+I_{3.1 .2}+I_{3.1 .3} \quad \text { (say). }
\end{aligned}
$$

Now consider,

$$
\begin{aligned}
I_{3.1 .1} & =O\left(\int_{0}^{\frac{1}{n+1}} \frac{e^{-\frac{\lambda}{2} t^{2} \log (n+1)}}{t}|\psi(t)| d t\right) \\
& =O\left[\int_{0}^{\frac{1}{n+1}}\left\{\frac{t \psi(t)}{t^{\alpha}}\right\}^{r} d t\right]^{\frac{1}{r}}\left[\int_{0}^{\frac{1}{n+1}}\left\{t^{\alpha-2} e^{-\frac{\lambda}{2} t^{2} \log (n+1)}\right\}^{s} d t\right]^{\frac{1}{s}} .
\end{aligned}
$$


Using second mean value theorem for integrals,

$$
\begin{aligned}
I_{3.1 .1} & =O\left\{\frac{e^{-\frac{\lambda}{2} \frac{\log (n+1)}{(n+1)^{2}}}}{(n+1)}\right\}\left[\int_{\epsilon}^{\frac{1}{n+1}}\left(t^{\alpha-2}\right)^{s} d t\right]^{\frac{1}{s}} \text { for } 0<\epsilon<\frac{1}{n+1} \\
& =O\left\{\frac{e^{-\frac{\lambda}{2} \frac{\log (n+1)}{(n+1)^{2}}}}{(n+1)}\right\}\left[\left\{\frac{t^{\alpha \alpha-2 s+1}}{s \alpha-2 s+1}\right\}_{\epsilon}^{\left.\frac{1}{n+1}\right]^{\frac{1}{s}}}\right. \\
& =O\left(\frac{1}{n+1}\right)\left[\frac{1}{(n+1)^{\alpha s-2 s+1}}\right]^{\frac{1}{s}} \\
& =O\left(\frac{1}{n+1}\right)\left[\frac{1}{(n+1)^{\alpha-2+\frac{1}{s}}}\right] \\
& =O\left(\frac{1}{n+1}\right)\left[\frac{1}{(n+1)^{\alpha-1-\frac{1}{r}}}\right] \text { since } \frac{1}{r}+\frac{1}{s}=1 \\
& =O\left[\frac{1}{(n+1)^{\alpha-\frac{1}{r}}}\right] .
\end{aligned}
$$

Now we consider,

$$
I_{3.1 .2}=O\{\lambda \log (n+1)\} \int_{0}^{\frac{1}{n+1}}|\sin (\lambda \log (n+1) \sin t) \| \psi(t)| d t .
$$

Since, for $0<t<\frac{1}{n+1}, \sin n t \leq n t$,

$$
I_{3.1 .2}=O\{\lambda \log (n+1)\} \int_{0}^{\frac{1}{n+1}} t|\psi(t)| d t .
$$

Using Hölder's inequality and Lemma 4,

$$
\begin{aligned}
I_{3.1 .2} & =O\{\lambda \log (n+1)\}\left[\int_{0}^{\frac{1}{n+1}}\left\{\frac{t \psi(t)}{t^{\alpha}}\right\}^{r} d t\right]^{\frac{1}{r}}\left[\int_{0}^{\frac{1}{n+1}}\left(t^{\alpha}\right)^{s} d t\right]^{\frac{1}{s}} \\
& =O\{\lambda \log (n+1)\}\left(\frac{1}{n+1}\right)\left[\left\{\frac{t^{\alpha+1}}{\alpha s+1}\right\}_{0}^{\frac{1}{n+1}}\right]^{\frac{1}{s}} \\
& =O\{\lambda \log (n+1)\}\left(\frac{1}{n+1}\right)\left[\frac{1}{(n+1)^{\alpha+1}}\right]^{\frac{1}{s}} \\
& =O\{\lambda \log (n+1)\}\left(\frac{1}{n+1}\right)\left[\frac{1}{(n+1)^{\alpha+\frac{1}{s}}}\right] \\
& =O\{\log (n+1)\}\left(\frac{1}{n+1}\right)\left[\frac{1}{(n+1)} \frac{1}{\alpha+1-\left(1-\frac{1}{s}\right)}\right] \\
& =O\{\log (n+1)\}\left(\frac{1}{n+1}\right)\left[\frac{1}{(n+1)^{\alpha+1-\frac{1}{r}}}\right] \text { since } \frac{1}{r}+\frac{1}{s}=1 \\
& =O\left\{\frac{\log (n+1)}{(n+1)^{2}}\right\}\left[\frac{1}{(n+1)^{\alpha-\frac{1}{r}}}\right] .
\end{aligned}
$$


Next we consider,

$$
\begin{aligned}
& I_{3.1 .3}=O \int_{0}^{\frac{1}{n+1}} e^{-\frac{\lambda}{2} t^{2} \log (n+1)}|\sin (t / 2) \| \psi(t)| d t \\
& =O\left\{\int_{0}^{\frac{1}{n+1}} t|\psi(t)| d t\right\} \\
& =O\left[\int_{0}^{\frac{1}{n+1}}\left\{\frac{t \psi(t)}{t^{\alpha}}\right\}^{r} d t\right]^{\frac{1}{r}}\left[\int_{0}^{\frac{1}{n+1}}\left(t^{\alpha}\right)^{s} d t\right]^{\frac{1}{s}} \\
& =O\left(\frac{1}{n+1}\right)\left[\left\{\frac{t^{\alpha s+1}}{\alpha s+1}\right\}_{0}^{\frac{1}{n+1}}\right]^{\frac{1}{s}} \\
& =O\left(\frac{1}{n+1}\right)\left[\frac{1}{(n+1)^{\alpha s+1}}\right]^{\frac{1}{s}} \\
& =O\left(\frac{1}{n+1}\right)\left[\frac{1}{(n+1)^{\alpha+\frac{1}{s}}}\right] \\
& =O\left(\frac{1}{n+1}\right)\left[\frac{1}{(n+1)^{\alpha+1-\left(1-\frac{1}{s}\right)}}\right] \\
& =O\left\{\frac{1}{(n+1)^{2}}\right\}\left[\frac{1}{(n+1)^{\alpha-\frac{1}{r}}}\right] \text { since } \frac{1}{r}+\frac{1}{s}=1 \text {. }
\end{aligned}
$$

Combining (5.9)-(5.12),

$$
\begin{aligned}
I_{3.1}= & O\left[\frac{1}{(n+1)^{\alpha-\frac{1}{r}}}\right]+O\left\{\frac{\log (n+1)}{(n+1)^{2}}\right\}\left[\frac{1}{(n+1)^{\alpha-\frac{1}{r}}}\right] \\
& +O\left\{\frac{1}{(n+1)^{2}}\right\}\left[\frac{1}{(n+1)^{\alpha-\frac{1}{r}}}\right] \\
= & O\left[\frac{\log (n+1) e}{(n+1)^{2}(n+1)^{\alpha-\frac{1}{r}}}\right]+O\left[\frac{1}{(n+1)^{\alpha-\frac{1}{r}}}\right] .
\end{aligned}
$$

Since, for $\frac{1}{n+1}<t<\pi,\left|\sin \left(\frac{t}{2}\right)\right| \geq \frac{t}{\pi}$

$$
\begin{aligned}
\tilde{K}_{n}(t) & =O\left[\frac{1}{\Gamma(\lambda+n) \sin \left(\frac{t}{2}\right)}\right] \\
& =O\left[\frac{1}{\Gamma(\lambda+n) t}\right] .
\end{aligned}
$$


Next we consider,

$$
I_{3.2} \leq \int_{\frac{1}{n+1}}^{\pi}|\psi(t)|\left|\tilde{K}_{n}(t)\right| d t
$$

Using Hölder's inequality, (5.14) and Lemma 4,

$$
\begin{aligned}
I_{3.2} & =O\left(\frac{1}{\Gamma(\lambda+n)}\right)\left[\int_{\frac{1}{n+1}}^{\pi}\left\{\frac{t^{-\delta} \psi(t)}{t^{\alpha}}\right\}^{r} d t\right]^{\frac{1}{r}}\left[\int_{\frac{1}{n+1}}^{\pi}\left(\frac{t^{\delta+\alpha}}{t}\right)^{s} d t\right]^{\frac{1}{s}} \\
& =O\left(\frac{1}{\Gamma(\lambda+n)}\right) \frac{1}{(n+1)^{-\delta}}\left[\int_{\frac{1}{n+1}}^{\pi} t^{(\delta+\alpha-1) s} d t\right]^{\frac{1}{s}} \\
& =O\left(\frac{1}{\Gamma(\lambda+n)}\right) \frac{1}{(n+1)^{-\delta}}\left[\left\{\frac{t^{(\delta+\alpha-1) s+1}}{(\delta+\alpha-1) s+1}\right\}^{\pi} \frac{1}{n+1}\right]^{\frac{1}{s}} \\
& =O\left(\frac{1}{\Gamma(\lambda+n)}\right) \frac{1}{(n+1)^{-\delta}}\left[\frac{1}{(n+1)^{(\delta+\alpha-1) s+1}}\right]^{\frac{1}{s}}\left[\frac{1}{(n+1)^{(\delta+\alpha-1)+\frac{1}{s}}}\right]^{-\frac{1}{(\lambda+1)^{-\delta}}}\left[\frac{1}{(n+1)^{\alpha-1+\frac{1}{s}}}\right] \\
& =O\left(\frac{1}{\Gamma(\lambda+n)}\right)\left[\frac{1}{(n+1)^{\alpha-\frac{1}{r}}}\right] \text { since } \frac{1}{\mathrm{r}}+\frac{1}{s}=1 .
\end{aligned}
$$

Collecting (5.8), (5.13) and (5.15),

$$
\begin{aligned}
\tilde{S}_{m}-\tilde{f}(x)= & O\left[\frac{\log (n+1) e}{(n+1)^{2}(n+1)^{\alpha-\frac{1}{r}}}\right]+\left[\frac{1}{(n+1)^{\alpha-\frac{1}{r}}}\right] \\
& +O\left(\frac{1}{\Gamma(\lambda+n)}\right)\left\{\frac{1}{(n+1)^{\alpha-\frac{1}{r}}}\right\} \\
= & O\left[\frac{1}{(n+1)^{\alpha-\frac{1}{r}}}\left\{\frac{\log (n+1) e}{(n+1)}+\frac{1}{\Gamma(\lambda+n)}+1\right\}\right] .
\end{aligned}
$$

This completes the proof of Theorem 3. 


\subsection{Proof of Theorem 4}

Following the calculations of Theorem 3,

$$
\begin{aligned}
\tilde{S}_{m}(x)-\tilde{f}(x) & =\frac{\Gamma(\lambda)}{2 \pi}\left[\int_{0}^{\frac{1}{n+1}}+\int_{\frac{1}{n+1}}^{\pi}\right] \psi(t) \tilde{K}_{n}(t) d t \\
& =O\left(I_{4.1}\right)+O\left(I_{4.2}\right) .
\end{aligned}
$$

Now,

$$
I_{4.1}=O\left[\int_{0}^{\frac{1}{n+1}}|\psi(t)|\left|K_{n}(t)\right| d t\right] .
$$

Using Lemma 3,

$$
\begin{aligned}
I_{4.1}= & O\left(\int_{0}^{\frac{1}{n+1}} \frac{e^{-\frac{\lambda}{2} t^{2} \log (n+1)}}{t}|\psi(t)| d t\right) \\
+ & O\{\lambda \log (n+1)\} \int_{0}^{\frac{1}{n+1}}|\sin (\lambda \log (n+1) \sin t) \| \psi(t)| d t \\
+ & O\left[\int_{0}^{\frac{1}{n+1}} e^{-\frac{\lambda}{2} t^{2} \log (n+1)}|\sin (t / 2) \| \psi(t)| d t\right] \\
=I_{4.1 .1}+I_{4.1 .2}+I_{4.1 .3} & \text { (say). }
\end{aligned}
$$

Using Minkowiski's inequality, we have a fact that $f L W\left(L_{r}, \xi(t)\right) \Rightarrow \psi L W\left(L_{r}, \xi(t)\right)$. Now we consider,

$$
\begin{aligned}
I_{4.1 .1} & =O\left(\int_{0}^{\frac{1}{n+1}} \frac{e^{-\frac{\lambda}{2} t^{2} \log (n+1)}}{t}|\psi(t)| d t\right) \\
& =O\left[\int_{0}^{\frac{1}{n+1}}\left\{\frac{t \psi(t) \sin ^{\beta}(t)}{\xi(t)}\right\}^{r} d t\right]^{\frac{1}{r}}\left[\int_{0}^{\frac{1}{n+1}}\left\{\frac{\xi(t) e^{-\frac{\lambda}{2} t^{2} \log (n+1)}}{\sin ^{\beta}(t)}\right]^{s}\right]^{\frac{1}{s}} d t \\
& =O\left\{e^{-\frac{\lambda}{2}} \frac{\log (n+1)}{(n+1)^{2}}\right\} O\left(\frac{1}{n+1}\right)\left[\int_{0}^{\frac{1}{n+1}}\left(\frac{\xi(t)}{\sin ^{\beta}(t)}\right)^{s} d t\right]^{\frac{1}{s}} \text { by (3.4) } \\
& =O\left(\frac{1}{n+1}\right)\left[\int_{0}^{\frac{1}{n+1}}\left(\frac{\xi(t)}{\sin ^{\beta}(t)}\right)^{s} d t\right]^{\frac{1}{s}} \\
& =O\left(\frac{1}{n+1}\right)\left[\int_{0}^{\frac{1}{n+1}}\left(\frac{\xi(t)}{t^{\beta}}\right)^{s} d t\right]^{\frac{1}{s}} \operatorname{since~} \sin t \geq \frac{2 t}{\pi} .
\end{aligned}
$$


Since $\xi(t)$ is a positive increasing function and using second mean value theorem for integrals,

$$
\begin{aligned}
I_{4.1 .1}= & O\left\{\left(\frac{1}{n+1}\right) \xi\left(\frac{1}{n+1}\right)\right\}\left[\int_{\epsilon} \frac{1}{n+1}\left\{\frac{1}{t^{\beta s}}\right\} d t\right]^{\frac{1}{s}} \text { for some } 0<\epsilon<\frac{1}{n+1} \\
= & O\left\{\left(\frac{1}{n+1}\right) \xi\left(\frac{1}{n+1}\right)\right\}\left[\left\{\frac{t^{-\beta s+1}}{-\beta s+1}\right\}_{\epsilon}^{\frac{1}{n+1}}\right]^{\frac{1}{s}} \\
= & O\left[\left\{\left(\frac{1}{n+1}\right) \xi\left(\frac{1}{n+1}\right)\right\}\left\{(n+1)^{\beta-1+\left(1-\frac{1}{s}\right)}\right\}\right] \\
= & O\left[\left\{\frac{1}{(n+1)^{2}} \xi\left(\frac{1}{n+1}\right)\right\}\left\{(n+1)^{\beta+\frac{1}{r}}\right\}\right] \text { since } \frac{1}{\mathrm{r}}+\frac{1}{s}=1 .
\end{aligned}
$$

Now,

$$
I_{4.1 .2}=O\{\lambda \log (n+1)\} \int_{0}^{\frac{1}{n+1}}|\sin (\lambda \log (n+1) \sin t) \| \psi(t)| d t .
$$

Since for $0<t<\frac{1}{n+1}$, sin $n t \leq n t$,

$$
I_{4.1 .2}=O\{\lambda \log (n+1)\} \int_{0}^{\frac{1}{n+1}} t|\psi(t)| d t .
$$

Hölder's inequality and the fact that $\psi(t) \in W\left(L_{r}, \xi(t)\right)$,

$$
\begin{aligned}
I_{4.1 .2} & =O\{\lambda \log (n+1)\}\left[\int_{0}^{\frac{1}{n+1}}\left\{\frac{t|\psi(t)| \sin ^{\beta}(t)}{\xi(t)}\right\}^{r} d t\right]^{\frac{1}{r}}\left[\int_{0}^{\frac{1}{n+1}}\left\{\frac{\xi(t)}{\sin ^{\beta} t}\right\}^{s} d t\right]^{\frac{1}{s}} \\
& =O\{\log (n+1)\} O\left(\frac{1}{n+1}\right)\left[\int_{0}^{\frac{1}{n+1}}\left\{\frac{\xi(t)}{\sin ^{\beta} t}\right\}^{s} d t\right]^{\frac{1}{s}} \text { by (3.4) } \\
= & O\left(\frac{\log (n+1)}{n+1}\right)\left[\int_{0}^{\frac{1}{n+1}}\left\{\frac{\xi(t)}{t^{\beta}}\right\}^{s} d t\right]^{\frac{1}{s}} \text { since } \sin t \geq 2 t / \pi .
\end{aligned}
$$

Since $\xi(t)$ is a positive increasing function and using second mean value theorem for integrals, 


$$
\begin{aligned}
I_{4.1 .2} & =O\left\{\left(\frac{\log (n+1)}{n+1}\right) \xi\left(\frac{1}{n+1}\right)\right\}\left[\int_{\epsilon}^{\frac{1}{n+1}}\left\{\frac{1}{t^{\beta s}}\right\} d t\right]^{\frac{1}{s}} \text { for some }<\epsilon<\frac{1}{n+1} \\
& =O\left\{\left(\frac{\log (n+1)}{n+1}\right) \xi\left(\frac{1}{n+1}\right)\right\}\left[\left\{\frac{t^{-\beta s+1}}{-\beta s+1}\right\}_{\epsilon}^{\frac{1}{n+1}}\right]^{\frac{1}{s}} \\
& =O\left[\left\{\left(\frac{\log (n+1)}{n+1}\right) \xi\left(\frac{1}{n+1}\right)\right\}\left\{(n+1)^{\beta-1+\left(1-\frac{1}{s}\right)}\right\}\right] \\
& =O\left[\left\{\frac{\log (n+1)}{(n+1)^{2}} \xi\left(\frac{1}{n+1}\right)\right\}\left\{(n+1)^{\beta+\frac{1}{r}}\right\}\right] \text { since } \frac{1}{r}+\frac{1}{s}=1 .
\end{aligned}
$$

Next we consider,

$$
\begin{aligned}
I_{4.1 .3}= & O\left[\int_{0}^{\frac{1}{n+1}} e^{-\frac{\lambda}{2} t^{2} \log (n+1)}\left|\sin \frac{t}{2}\right||\phi(t)| d t\right] \\
= & O\left[\int_{0}^{\frac{1}{n+1}} t|\psi(t)| d t\right] .
\end{aligned}
$$

Using Hölder's inequality and the fact that $\psi(t) \in W\left(L_{r} \xi(t)\right)$,

$$
\begin{aligned}
I_{4.1 .3} & =O\left[\int_{0}^{\frac{1}{n+1}}\left\{\frac{t|\psi(t)| \sin ^{\beta}(t)}{\xi(t)}\right\}^{r} d t\right]^{\frac{1}{r}}\left[\int_{0}^{\frac{1}{n+1}}\left\{\frac{\xi(t)}{\sin ^{\beta} t}\right\}^{s} d t\right]^{\frac{1}{s}} \\
& =O\left(\frac{1}{n+1}\right)\left[\int_{0}^{\frac{1}{n+1}}\left\{\frac{\xi(t)}{\sin ^{\beta} t}\right\}^{s} d t\right]^{\frac{1}{s}} \text { by (3.4) }
\end{aligned}
$$

Since, $\sin t \geq 2 t / \pi$,

$$
I_{4.1 .3}=O\left(\frac{1}{n+1}\right)\left[\int_{0}^{\frac{1}{n+1}}\left\{\frac{\xi(t)}{t^{\beta}}\right\}^{s} d t\right]^{\frac{1}{s}} .
$$

Since $\xi(t)$ is a positive increasing function and using second mean value theorem for integrals, 


$$
\begin{aligned}
I_{4.1 .3}=O\left\{\left(\frac{1}{n+1}\right) \xi\left(\frac{1}{n+1}\right)\right\}\left[\int_{\epsilon}^{\frac{1}{n+1}}\left\{\frac{1}{t^{\beta s}}\right\} d t\right]^{\frac{1}{s}} \\
=O\left\{\left(\frac{1}{n+1}\right) \xi\left(\frac{1}{n+1}\right)\right\}\left[\left\{\frac{t^{-\beta s+1}}{-\beta s+1}\right\}_{\epsilon}^{\frac{1}{n+1}}\right]^{\frac{1}{s}} \\
=O\left[\left\{\left(\frac{1}{n+1}\right) \xi\left(\frac{1}{n+1}\right)\right\}\left\{(n+1)^{\beta-\frac{1}{s}}\right\}\right] \\
=O\left[\left\{\left(\frac{1}{n+1}\right) \xi\left(\frac{1}{n+1}\right)\right\}\left\{(n+1)^{\beta-1+\left(1-\frac{1}{s}\right)}\right\}\right] \\
=O\left[\left\{\frac{1}{(n+1)^{2}} \xi\left(\frac{1}{n+1}\right)\right\}\left\{(n+1)^{\beta+\frac{1}{r}}\right\}\right] \text { since } \frac{1}{r}+\frac{1}{s}=1 .
\end{aligned}
$$

Combining from (5.17) to (5.20),

$$
\begin{aligned}
I_{4.1}= & O\left[\left\{\frac{1}{(n+1)^{2}} \xi\left(\frac{1}{n+1}\right)\right\}\left\{(n+1)^{\beta+\frac{1}{r}}\right\}\right] \\
& +O\left[\left\{\frac{\log (n+1)}{(n+1)^{2}} \xi\left(\frac{1}{n+1}\right)\right\}\left\{(n+1)^{\beta+\frac{1}{r}}\right\}\right] \\
& +O\left[\left\{\frac{1}{(n+1)^{2}} \xi\left(\frac{1}{n+1}\right)\right\}\left\{(n+1)^{\beta+\frac{1}{r}}\right\}\right] .
\end{aligned}
$$

Using Hölder's inequality, $|\sin t| \leq 1, \sin t \geq 2 t / \pi$, conditions (3.3), (3.5) and second mean value theorem for integrals and the fact $\psi(t) \in W\left(L_{r}, \xi(t)\right)$,

$$
\begin{aligned}
I_{4.2} & =O\left(\int_{\frac{1}{n+1}}^{\pi} \frac{1}{\Gamma(\lambda+n) t}|\psi(t)| d t\right) \\
& =O\left(\frac{1}{\Gamma(\lambda+n)}\right)\left[\int_{\frac{1}{n+1}}^{\pi}\left\{\frac{t^{-\delta}|\psi(t)| \sin ^{\beta}(t)}{\xi(t)}\right\}^{r} d t\right]^{\frac{1}{r}}\left[\int_{\left.\frac{1}{n+1}\left\{\frac{\xi(t)}{t^{1-\delta} \sin ^{\beta} t}\right\}^{s} d t\right]^{\frac{1}{s}}}\right. \\
& =O\left(\frac{1}{\Gamma(\lambda+n)}\right)\left[\int_{\frac{1}{n+1}}^{\pi}\left\{\frac{t^{-\delta}|\psi(t)|}{\xi(t)}\right\}^{r} d t\right]^{\frac{1}{r}}\left[\int_{\frac{1}{n+1}}^{\pi}\left\{\frac{\xi(t)}{t^{1-\delta} \sin ^{\beta} t}\right\}^{s} d t\right]^{\frac{1}{s}} \\
& =O\left[\left\{\frac{1}{\Gamma(\lambda+n)}\right\}\left\{(n+1)^{\delta}\right\}\right]\left[\int_{\frac{1}{n+1}}^{\pi}\left\{\frac{\xi(t)}{t^{1-\delta} \sin ^{\beta} t}\right\}^{s} d t\right]^{\frac{1}{s}} \\
& =O\left[\left\{\frac{1}{\Gamma(\lambda+n)}\right\}\left\{(n+1)^{\delta}\right\}\right]\left[\int_{\frac{1}{n+1}}^{\pi}\left\{\frac{\xi(t)}{t^{-\delta+\beta+1}}\right\}^{s} d t\right]^{\frac{1}{s}} .
\end{aligned}
$$


Putting $t=\frac{1}{y}$

$$
\begin{aligned}
I_{4.2} & =O\left\{\frac{(n+1)^{\delta}}{\Gamma(\lambda+n)}\right\}\left[\int_{\pi}^{n+1}\left\{\frac{\xi\left(\frac{1}{y}\right)}{\gamma^{\delta-\beta-1^{\prime}}}\right\} \frac{d y}{\gamma^{2}}\right]^{\frac{1}{s}} \\
& =O\left\{\frac{(n+1)^{\delta}}{\Gamma(\lambda+n)} \xi\left(\frac{1}{n+1}\right)\right\}\left[\int_{\eta}^{n+1}\left\{\frac{d y}{\gamma^{s(\delta-\beta-1)+2}}\right\} d t\right]^{\frac{1}{s}} \text { for some } \frac{1}{\pi} \leq \eta \leq n+1 \\
& =O\left\{\frac{(n+1)^{\delta}}{\Gamma(\lambda+n)} \xi\left(\frac{1}{n+1}\right)\right\}\left[\int_{1}^{n+1}\left\{\frac{d y}{\gamma^{s(\delta-\beta-1)+2}}\right\} d t\right]^{\frac{1}{s}} \text { for some } \frac{1}{\pi} \leq 1 \leq n+1 \\
& =O\left\{\frac{(n+1)^{\delta}}{\Gamma(\lambda+n)} \xi\left(\frac{1}{n+1}\right)\right\}\left[\left\{\frac{y^{s(\beta+1-\delta)-1}}{s(\beta+1-\delta)-1}\right\}_{1}^{n+1}\right]^{\frac{1}{s}} \\
& =O\left\{\frac{(n+1)^{\delta}}{\Gamma(\lambda+n)} \xi\left(\frac{1}{n+1}\right)\right\}\left\{(n+1){ }^{1+\beta-\delta-\frac{1}{s}}\right\} \\
& =O\left\{\frac{\xi\left(\frac{1}{n+1}\right)}{\Gamma(\lambda+n)}\right\}\left\{(n+1)^{\beta+\frac{1}{r}}\right\} \text { since } \frac{1}{\mathrm{r}}+\frac{1}{s}=1 .
\end{aligned}
$$

Combining from (5.16), (5.21) and (5.22)

$$
\begin{aligned}
\left|S_{m}(x)-f(x)\right|= & O\left[\left\{\frac{1}{(n+1)^{2}} \xi\left(\frac{1}{n+1}\right)\right\}\left\{(n+1)^{\beta+\frac{1}{r}}\right\}\right] \\
& +O\left[\left\{\frac{\log (n+1)}{(n+1)^{2}} \xi\left(\frac{1}{n+1}\right)\right\}\left\{(n+1)^{\beta+\frac{1}{r}}\right\}\right] \\
& +O\left[\left\{\frac{1}{(n+1)^{2}} \xi\left(\frac{1}{n+1}\right)\right\}\left\{(n+1)^{\beta+\frac{1}{r}}\right\}\right] \\
& +O\left\{\frac{\xi\left(\frac{1}{n+1}\right)}{\Gamma(\lambda+n)}\right\}\left\{(n+1)^{\beta+\frac{1}{r}}\right\} \\
= & O\left\{(n+1)^{\beta+\frac{1}{r}} \xi\left(\frac{1}{n+1}\right)\right\}\left[\frac{2}{(n+1)^{2}}+\frac{\log (n+1)}{(n+1)^{2}}+\frac{1}{\Gamma(\lambda+n)}\right] .
\end{aligned}
$$

Now using $\mathrm{L}_{\mathrm{r}}$-norm, we get

$$
\begin{aligned}
\left\|S_{m}(x)-f(x)\right\|= & \left\{\int_{0}^{2 \pi}\left|S_{m}(x)-f(x)\right|^{r} d x\right\}^{\frac{1}{r}} \\
= & O\left[\int_{0}^{2 \pi}(n+1)^{\beta+\frac{1}{r}} \xi\left(\frac{1}{n+1}\right)\right. \\
& \left.\cdot\left\{\frac{2}{(n+1)^{2}}+\frac{\log (n+1)}{(n+1)^{2}}+\frac{1}{\Gamma(\lambda+n)}\right\} d x\right]^{\frac{1}{r}} \\
= & {\left[\left\{(n+1)^{\beta+\frac{1}{r}} \xi\left(\frac{1}{n+1}\right)\right\}\right.} \\
& \left.\cdot\left\{\frac{2}{(n+1)^{2}}+\frac{\log (n+1)}{(n+1)^{2}}+\frac{1}{\Gamma(\lambda+n)}\right\}\right]\left[\left\{\int_{0}^{2 \pi} d x\right\}^{\frac{1}{r}}\right] \\
=O & \left\{(n+1)^{\beta+\frac{1}{r}} \xi\left(\frac{1}{n+1}\right)\right\}\left[\frac{2}{(n+1)^{2}}+\frac{\log (n+1)}{(n+1)^{2}}+\frac{1}{\Gamma(\lambda+n)}\right] \\
= & O\left\{(n+1)^{\beta+\frac{1}{r}} \xi\left(\frac{1}{n+1}\right)\right\}\left[1+\frac{\log (n+1) e}{(n+1)^{2}}+\frac{1}{\Gamma(\lambda+n)}\right] .
\end{aligned}
$$


This completes the proof of Theorem 4 .

\section{Authors' contributions}

HK framed the problems. HK and KS carried out the results and wrote the manuscripts. All the authors read and approved the final manuscripts.

\section{Competing interests}

The authors declare that they have no competing interests.

Received: 25 January 2011 Accepted: 12 October 2011 Published: 12 October 2011

\section{References}

1. Karamata, J: Theorems surla sommabilite exponentielle etd autres Sommabilities sattachant. Math (Cliy). 9, 164 (1935)

2. Lotosky, AV: On a linear transformation of sequences (in Russian). Ivanov Gos Red Inst Uchen Zap. 4, 61 (1963)

3. Agnew, RP: The Lotosky method for evaluation of series. Michigan Math J. 4, 105 (1957)

4. Vuckkoviĉ, V: The summability of Fourier series by Karamata method. Maths Zeitchr. 89, 192 (1965). doi:10.1007/ BF02116860

5. Kathal, PD: A new criteria for Karamata summability of Fourier series. Riv Math Univ Parma Italy. 10, 33-38 (1969)

6. Ojha, AK: pp. 120-126. Ph.D. Thesis, B.H.U (1982)

7. Tripathi, LM, Lal, S: $K^{\lambda}$-summability of Fourier series. Jour Sci Res. 34, 69-74 (1984)

8. Alexits, G: Convergence problems of orthogonal series. Translated from German by I Folder. International series of Monograms in Pure and Applied Mathematics. 20 (1961)

9. Sahney, BN, Goel, DS: On the degree of continuous functions. Ranchi Univ Math J. 4, 50-53 (1973)

10. Chandra, P: Trigonometric approximation of functions in $L^{p}$ norm. J Math Anal Appl. 275(1), 13-26 (2002). doi:10.1016/ S0022-247X(02)00211-1

11. Qureshi, K: On the degree of approximation of a periodic function $f$ by almost Nörlund means. Tamkang J Math. 12(1), 35-38 (1981)

12. Qureshi, K, Neha, HK: A class of functions and their degree of approximation. Ganita. 41(1), $37-42$ (1990)

13. Rhoades, BE: On the degree of approximation of functions belonging to Lipschitz class by Hausdorff means of its Fourier series. Tamkang J Math. 34(3), 245-247 (2003)

14. Zygmund, A: Trigonometric Series. Cambridge University Press, Cambridge (1939)

15. McFadden, L: Absolute Nörlund summability. Duke Math J. 9, 168-207 (1942). doi:10.1215/50012-7094-42-00913-X

16. Titchmarsh, EC: The Theory of Functions. Oxford University Press, Oxford (1939)

doi:10.1186/1029-242X-2011-85

Cite this article as: Nigam and Sharma: A study on degree of approximation by Karamata summability method. Journal of Inequalities and Applications 2011 2011:85.

\section{Submit your manuscript to a SpringerOpen ${ }^{\odot}$ journal and benefit from:}

- Convenient online submission

- Rigorous peer review

- Immediate publication on acceptance

- Open access: articles freely available online

- High visibility within the field

- Retaining the copyright to your article

Submit your next manuscript at $\boldsymbol{s p r i n g e r o p e n . c o m ~}$ 